\title{
Rheodynamic lubrication of a squeeze film bearing under sinusoidal squeeze motion
}

\author{
A. KANDASAMY* ${ }^{*}$ and K.P. VISHWANATH** \\ Department of Mathematical and Computational Sciences \\ National Institute of Technology Karnataka, Surathkal, Mangalore - 575.025, India \\ E-mails: kandy_aks@yahoo.com / shastry_vishwanath@yahoo.co.in
}

\begin{abstract}
Lubricants with variable viscosity are assuming importance for their applications in polymer industry, thermal reactors and in biomechanics. With the bearing operations in machines being subjected to high speeds, loads, increasing mechanical shearing forces and continually increasing pressures, there has been an increasing interest to use non-Newtonian fluids characterized by an yield value. The most elementary constitutive equation in common use that describes a material which yields is that of Bingham fluid. In the present work, the problem of a circular squeeze film bearing lubricated with Bingham fluid under the sinusoidal squeeze motion has been analyzed. The shape and extent of the core for the case of sinusoidal squeeze motion has been determined numerically for various values of the Bingham number. Numerical solutions have been obtained for the bearing performances such as pressure distribution and load capacity for different values of Bingham number, Reynolds number and for various amplitudes of squeeze motion. The effects of fluid inertia, non-Newtonian characteristics, and the amplitudes of squeeze motion on the bearing performances have been discussed.
\end{abstract}

Mathematical subject classification: 76A05, 76D08.

Key words: non-Newtonian fluid, Bingham fluid, yield stress, rheodynamic lubrication, squeeze film bearing, sinusoidal motion.

\#726/07. Received: 01/II/07. Accepted: 24/V/07.

* Professor and Head

** Research Scholar 


\section{Introduction}

Recently, it has been emphasized that in order to analyze the performance of bearings adequately, it is necessary to take into account the combined effects of fluid inertia and non-Newtonian characteristics of lubricants. In most squeeze film bearings, the effects of fluid inertia forces become significant with increase in squeeze velocity as well as film thickness.

The effects of fluid inertia forces and non-Newtonian characteristics of lubricants in the squeeze film bearings have been examined by several investigators, (Tichy and Winer [8], Covey and Stanmore [2], Gartling and Phan-Thien [4], Donovan and Tanner [7], Huang et al. [6], Usha and Vimala [9]) but there are few papers attempting to describe the combined effects of fluid inertia forces and non-Newtonian characteristics of lubricants (Elkough [3], Batra and Kandasamy [1]) . Even these works are without considering the sinusoidal motion of the squeeze film bearing. With sinusoidal squeeze motion, Usha and Vimala [10] have applied the energy integral approach to find the behavior of curved squeeze film bearing using a Newtonian lubricant. Hashimoto and Wada [5] have examined the effects of fluid inertia forces in a squeeze film bearing with sinusoidal motion lubricated using a power law fluid.

In the present work, the problem of a squeeze film bearing lubricated with Bingham fluid under the sinusoidal squeeze motion of Circular plates has been analyzed. During the operations of the bearings, the maximum viscous shearing stresses arise in the region between the plates. Therefore, there may be a region in the film where the shearing stresses do not exceed the yield value of the lubricant and thereby a core with zero velocity gradient is formed. The flow occurs only in the region where the shear stress exceeds the yield value. The shape and extent of the core has been determined numerically for various values of the Bingham number for the case of sinusoidal squeeze motion. The flow is confined to the region between the core and the circular plates of the bearing. Numerical solutions have been obtained for the bearing performances such as pressure distribution and load capacity for different values of Bingham number, Reynolds number and for various amplitudes of squeeze motion. The properties of the squeeze film are investigated through the inertial and non-Newtonian effects on the load capacity of the bearing for various sinusoidal conditions. 


\section{Mathematical formulation of the problem}

The geometry of the problem is as shown in Fig. 1. We consider an isothermal, incompressible, steady flow of a time independent Bingham fluid squeezed between two circular plates separated by a distance $h$. Let $2 R$ be the diameter of the bearings approaching each other with a squeeze velocity $v_{s}$ under a normal load $W$. We consider cylindrical polar co-ordinates $(r, \theta, z)$ with axial symmetry and the origin fixed at the center of the lower plate. Here $r$ represents the distance measured along the radial direction and $z$ along the axis normal to the plates. Let $v_{r}$ and $v_{z}$ represent velocity components in the radial $(r)$ and axial directions $(z)$ respectively. Let $p$ denote the pressure and $\rho$ the density of the fluid. It is assumed that there is no sliding motion of the two plates.

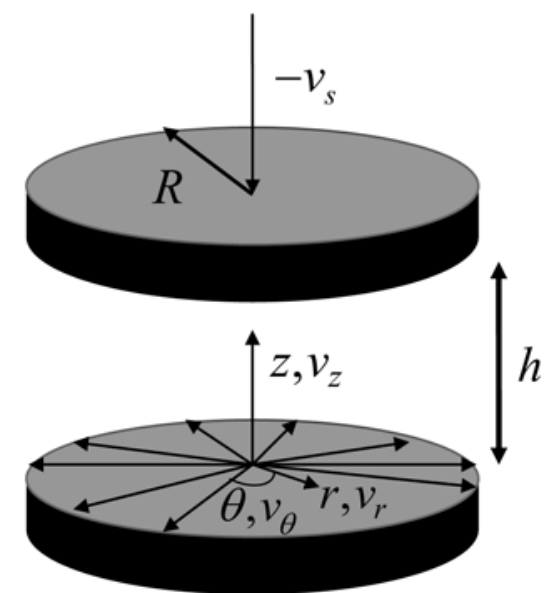

Figure 1 - Geometry of the squeeze film bearing.

The constitutive equation of a Bingham fluid is given by,

$$
\tau_{i j}=2\left(\eta_{1}+\frac{\eta_{2}}{I^{\frac{1}{2}}}\right) e_{i j}, \quad\left(\frac{1}{2} \tau_{i j} \tau_{i j} \geq \eta_{2}^{2}\right)
$$

where $\tau_{i j}$ are the deviatoric stress components, $\eta_{1}$ and $\eta_{2}$ are constants named the plastic viscosity and yield value respectively, $e_{i j}$ represents the rate of deformation components and $I=2 e_{i j} e_{i j}$ is strain invariant.

In those regions of the film, where the shear stress is less than the yield value, there will be core formation, which will move with constant velocity $v_{c}$. Let the boundaries of the core be given by $z=h_{1}(r)$ and $z=h_{2}(r)$ as shown in Fig. 2. 


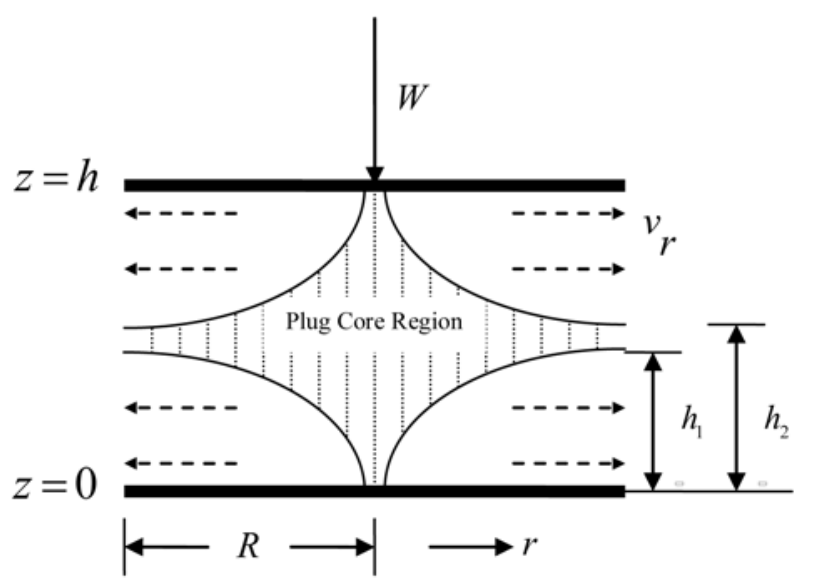

Figure 2 - Shape of the core.

Applying the basic assumptions of lubrication theory for thin films, the governing equations for the above squeeze film system, including inertia forces, is given by:

$$
\begin{aligned}
\rho\left(v_{r} \frac{\partial v_{r}}{\partial r}+v_{z} \frac{\partial v_{r}}{\partial z}\right) & =-\frac{\partial p}{\partial r}+\frac{\partial \tau_{r z}}{\partial z} \\
\frac{\partial p}{\partial z} & =0 \\
\frac{1}{r} \frac{\partial\left(r v_{r}\right)}{\partial r}+\frac{\partial v_{z}}{\partial z} & =0 \\
\tau_{r z} & =\eta_{2}+\eta_{1}\left(\frac{\partial v_{r}}{\partial z}\right)
\end{aligned}
$$

The above equations (2), (3) and (5) together with the continuity equation (4), are to be solved under the following boundary conditions:

$$
\begin{array}{rlrlrl}
v_{r} & =0 & \text { at } & z & =0, h \\
v_{z}=-v_{s} & \text { at } & z & =h \\
v_{z}=0 & \text { at } & z & =0 \\
p & =p_{a} & \text { at } & r & =R
\end{array}
$$

$v_{r}$ and $\frac{\partial v_{r}}{\partial z}$ are continuous at $z=h_{1}(r)$ and $z=h_{2}(r)$. Here $p_{a}$ is the atmospheric pressure. 


\section{Solution to the problem}

The integral form of the continuity equation, also called the equation of squeeze motion is given by,

$$
2 \pi r \int_{0}^{h} v_{r} d z=\pi r^{2} v_{s}
$$

Averaging the inertia terms in the momentum equation (2) by assuming it to be a constant over the film thickness (Hashimoto and Wada [5]), and performing integration by parts in the resulting equation using the continuity equation (4) and boundary conditions, we get,

$$
\frac{\rho}{h}\left[\frac{r}{2}\left(\frac{\partial v_{s}}{\partial t}\right)+\frac{\partial}{\partial r} \int_{0}^{h} v_{r}^{2} d z+\frac{1}{r} \int_{0}^{h} v_{r}^{2} d z\right]+\frac{d p}{d r}=\frac{\partial \tau_{r z}}{\partial z}
$$

Here, we introduce the following modified pressure gradient:

$$
f \equiv \frac{\rho}{h}\left[\frac{r}{2}\left(\frac{\partial v_{s}}{\partial t}\right)+\frac{\partial}{\partial r} \int_{0}^{h} v_{r}^{2} d z+\frac{1}{r} \int_{0}^{h} v_{r}^{2} d z\right]+\frac{d p}{d r}
$$

Hence, from equations (8) and (9), we have,

$$
\frac{\partial \tau_{r z}}{\partial z}=f
$$

As the modified pressure gradient is independent of the coordinate $z$, equation (10) can be integrated as follows:

$$
\tau_{r z}=f z+c_{1}
$$

Substituting $\tau_{r z}$ from equation (11) into equation (5) and integrating the resulting equations using the boundary conditions (6), we get the velocity distribution in the two flow regions separating the core region as,

$$
\begin{aligned}
& v_{r}=\frac{f}{\eta_{1}}\left(\frac{z^{2}}{2}-h_{1} z\right) \quad \text { in } 0 \leq z \leq h_{1}(r) \\
& v_{r}=\frac{f}{\eta_{1}}\left(\frac{z^{2}}{2}-\frac{h^{2}}{2}-h_{2} z+h_{2} h\right) \text { in } h_{2}(r) \leq z \leq h
\end{aligned}
$$

and the core velocity as,

$$
v_{r}=v_{c}=-\frac{f}{\eta_{1}}\left(\frac{h_{1}^{2}}{2}\right)=-\frac{f}{\eta_{1}}\left(\frac{\left(h-h_{2}\right)^{2}}{2}\right) \quad \text { in } \quad h_{1}(r) \leq z \leq h_{2}(r)
$$


From equation (14), we have,

$$
h_{1}(r)=h-h_{2}(r)
$$

Considering the equilibrium of an element of the core in the fluid, we get,

$$
f=-\frac{2 \eta_{2}}{H}
$$

where

$$
H=H(r)=h_{2}(r)-h_{1}(r)
$$

represents the thickness of the core.

Using the velocity equations (12), (13), (14) and the equation of squeeze motion (7), we get,

$$
f=-\frac{12 r \eta_{1} v_{s}}{H^{3}-3 h^{2} H+2 h^{3}}
$$

Eliminating $f$ from equations (16) and (18), we get an algebraic equation for determining the thickness of the core as,

$$
H^{3}-3\left[h^{2}-\frac{2 r \eta_{1} v_{s}}{\eta_{2}}\right] H+2 h^{3}=0
$$

Further, Substituting for $f$ from equation (18) in the velocity equations (12), (13) and (14), we get,

$$
\begin{aligned}
& v_{r}=\frac{6 r v_{s}(h-H-z) z}{(h-H)^{2}(2 h+H)} \text { in } 0 \leq z \leq h_{1}(r) \\
& v_{r}=\frac{3 r v_{s}}{2(2 h+H)} \text { in } h_{1}(r) \leq z \leq h_{2}(r) \\
& v_{r}=\frac{6 r v_{s}(h-z)(-H+z)}{(h-H)^{2}(2 h+H)} \text { in } h_{2}(r) \leq z \leq h
\end{aligned}
$$

Substituting $v_{r}$ from equations (12), (13) and (14) into equation (9), we obtain the following equation for pressure gradient:

$$
\begin{aligned}
\frac{d p}{d r}= & f-\frac{\rho r}{2 h}\left(\frac{\partial v_{s}}{\partial t}\right) \\
& -\frac{63 H^{2}+198 H h+144 h^{2}-21 r H H^{\prime}-6 r h H^{\prime}}{\left.20(H+2 h)^{3}\right)}\left(\frac{\rho v_{s}^{2} r}{h}\right)
\end{aligned}
$$


where

$$
H^{\prime}=\frac{d H}{d r}=\frac{d h_{2}}{d r}-\frac{d h_{1}}{d r}
$$

Let us introduce the sinusoidal squeeze motion on the film thickness as

$$
h=h_{0}+a \cos \left(w_{f} t\right)
$$

where $h_{0}$ is the mean film thickness, $a$ is amplitude of oscillation, $w_{f}$ is the frequency and $t$ is time of oscillation.

Then, the squeeze velocity and squeeze acceleration are given respectively, as,

$$
v_{s}=-\frac{\partial h}{\partial t}=a w_{f} \sin \left(w_{f} t\right)
$$

and

$$
\frac{\partial v_{s}}{\partial t}=a w_{f}^{2} \cos \left(w_{f} t\right)
$$

The following non-dimensional quantities are introduced:

$$
\begin{gathered}
A=\frac{a}{h_{0}}, \quad z^{*}=\frac{z}{h_{0}}, \quad h^{*}=\frac{h}{h_{0}}, \quad r^{*}=\frac{r}{R}, \quad v_{r}^{*}=\frac{v_{r}}{R w_{f}}, \\
H^{*}=\frac{H}{h_{0}}, \quad V_{s}^{*}=\frac{v_{s}}{h_{0} w_{f}}, \quad T=w_{f} t, \quad B=\frac{\eta_{2} h_{0}}{R w_{f} \eta_{1}}, \\
P=\frac{h_{0}^{2} p}{\eta_{1} w_{f} R^{2}}, \quad P_{a}=\frac{h_{0}^{2} p_{a}}{\eta_{1} w_{f} R^{2}}, \quad \Re=\frac{\rho h_{0}^{2} w_{f}}{\eta_{1}},
\end{gathered}
$$

where $B$ is called the Bingham number of the fluid and $\Re$ is the Reynolds number for the squeeze film.

Using the non-dimensional quantities stated above in the equations (25), (26) and (27), we get

$$
h^{*}=1+A \cos T \Longrightarrow V_{s}^{*}=-\frac{\partial h^{*}}{\partial T}=A \sin T \Longrightarrow \frac{\partial V_{s}^{*}}{\partial T}=A \cos T
$$

Then, the non-dimensional form of equation (19) can be expressed as

$$
\left(H^{*}\right)^{3}-3\left[(1+A \cos T)^{2}+\frac{2 A \sin T r^{*}}{B}\right] H^{*}+2(1+A \cos T)^{3}=0
$$

The core thickness is determined from the above algebraic equation. The root $H^{*}\left(r^{*}, B, A, T\right)$ of equation (30), which is positive and smaller than unity, determines the shape of the yield surface. The value of the root for a particular 
material for a given Bingham number can be obtained for various values of $r^{*}$, $A$ and $T$ using any numerical iterative technique.

Further, non-dimensional forms of velocity equations (20), (21) and (22) are given as follows:

$$
\begin{aligned}
& v_{r}^{*}=\frac{6 r^{*} V_{s}^{*}\left(h^{*}-H^{*}-z^{*}\right) z^{*}}{\left(h^{*}-H^{*}\right)^{2}\left(2 h^{*}+H^{*}\right)} \text { in } 0 \leq z^{*} \leq \frac{\left(h^{*}-H^{*}\right)}{2} \\
& v_{r}^{*}=\frac{3 r^{*} V_{s}^{*}}{2\left(2 h^{*}+H^{*}\right)} \text { in } \frac{\left(h^{*}-H^{*}\right)}{2} \leq z^{*} \leq \frac{\left(h^{*}+H^{*}\right)}{2} \\
& v_{r}^{*}=\frac{6 r^{*} V_{s}^{*}\left(h^{*}-z^{*}\right)\left(-H^{*}+z^{*}\right)}{\left(h^{*}-H^{*}\right)^{2}\left(2 h^{*}+H^{*}\right)} \text { in } \frac{\left(h^{*}+H^{*}\right)}{2} \leq z^{*} \leq h^{*}
\end{aligned}
$$

The velocity profiles along radial direction can be obtained by substituting the values of $r^{*}, h^{*}, z^{*}, A, T$ in the above equations.

Non-dimensionalising equation (23), and integrating it using the boundary conditions, we obtain the following expression for pressure distribution:

$$
\begin{aligned}
P-P_{a}= & \int_{1}^{r^{*}}\left[\frac{-12 r^{*} A \sin T}{\left(H^{*}\right)^{3}-3(1+A \cos T)^{2} H^{*}+2(1+A \cos T)^{3}}\right] d r^{*} \\
& -\int_{1}^{r^{*}}\left[\frac{\Re r^{*} A \cos T}{2(1+A \cos T)}\right] d r^{*}-\int_{1}^{r^{*}}\left[\frac{\Re r^{*}(A \sin T)^{2}}{20(1+A \cos T)}\right] \Gamma d r^{*}
\end{aligned}
$$

where

$$
=\begin{gathered}
\Gamma \equiv \Gamma\left(H^{*}, H^{* \prime}, A, T, r^{*}\right) \\
=\left[\frac{63 H^{*^{2}}+198(1+A \cos T) H^{*}+144(1+A \cos T)^{2}-21 r^{*} H^{*} H^{* \prime}-6 r^{*}(1+A \cos T) H^{* \prime}}{\left(H^{*}+2(1+A \cos T)\right)^{3}}\right]
\end{gathered}
$$

and

$$
H^{* \prime}=\frac{2 A \sin T H^{*}}{B\left\{\left(H^{*}\right)^{2}-\left[(1+A \cos T)^{2}+\frac{2 r^{*} A \sin T}{B}\right]\right\}}
$$

The pressure distribution of the squeeze film bearing can be determined for different values of Bingham number, squeeze Reynolds number, amplitude and time by integrating equation (34) numerically.

The load capacity of the circular squeeze film bearing is then given by,

$$
W=\int_{0}^{1}\left(P-P_{a}\right) r^{*} d r^{*}
$$


The integral in equation (37) can be evaluated numerically, for various values of squeeze Reynolds number, amplitude, time and for materials with different values of Bingham number.

\section{Results and Discussion}

The behavior of core thickness $\left(H^{*}\right)$ for various values of amplitude $(A)$, time $(T)$ and non-Newtonian characteristics $(B)$ at every point of radius $\left(r^{*}\right)$ is computed and the results are given in Figs. 3-6. The core thickness is maximum at the center of the plates and decreases towards the periphery. The core formation decreases with the increase of time for a constant Bingham number and amplitude. Further, it has been found that the thickness of the core increases when the Bingham number increases for a constant time value and amplitude.

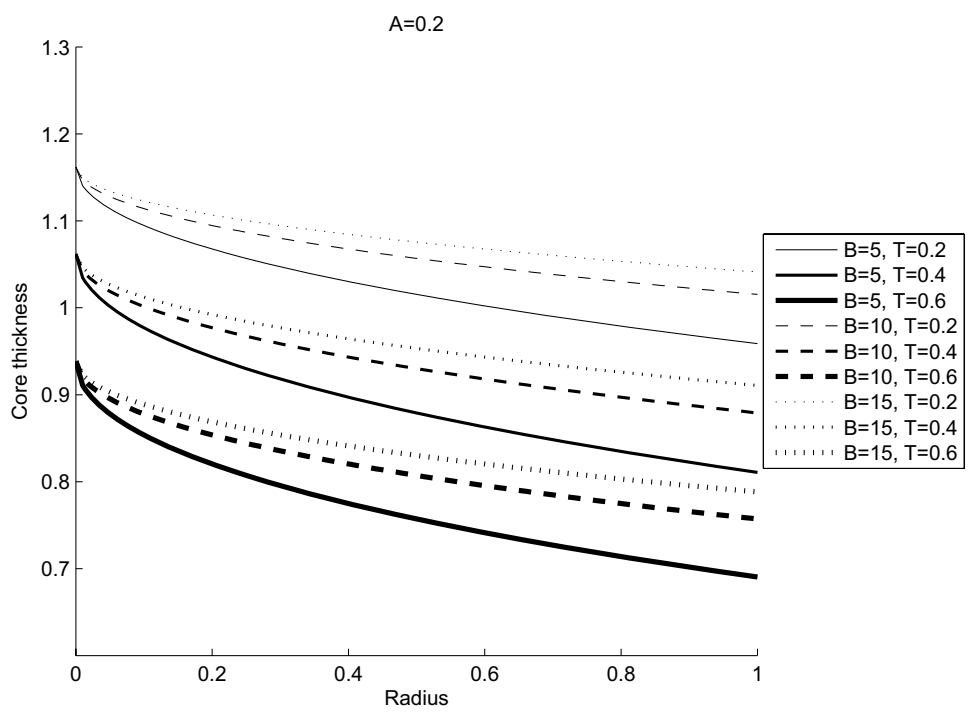

Figure 3 - Core thickness variation along the radius for $A=0.2$.

The velocity profiles along the radial direction $\left(r^{*}\right)$ have been plotted for various values along the axial direction $\left(z^{*}\right)$ at a constant amplitude for different time values and non-Newtonian characteristics in Figs. 7-11. In Figs. 7-9, the shape of the profile is observed to be the same for increase in time of oscillation although the maximum value of velocity is found to increase with the increase of time. In Figs. 9-11, the region of constant velocity in the curve is observed to be 


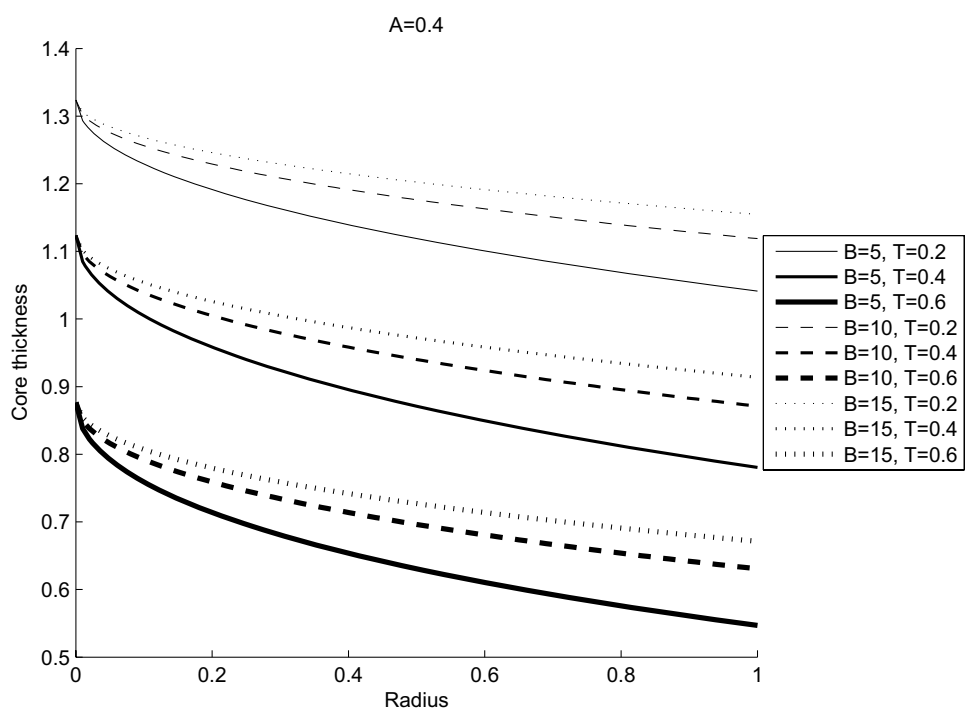

Figure 4 - Core thickness variation along the radius for $A=0.4$.

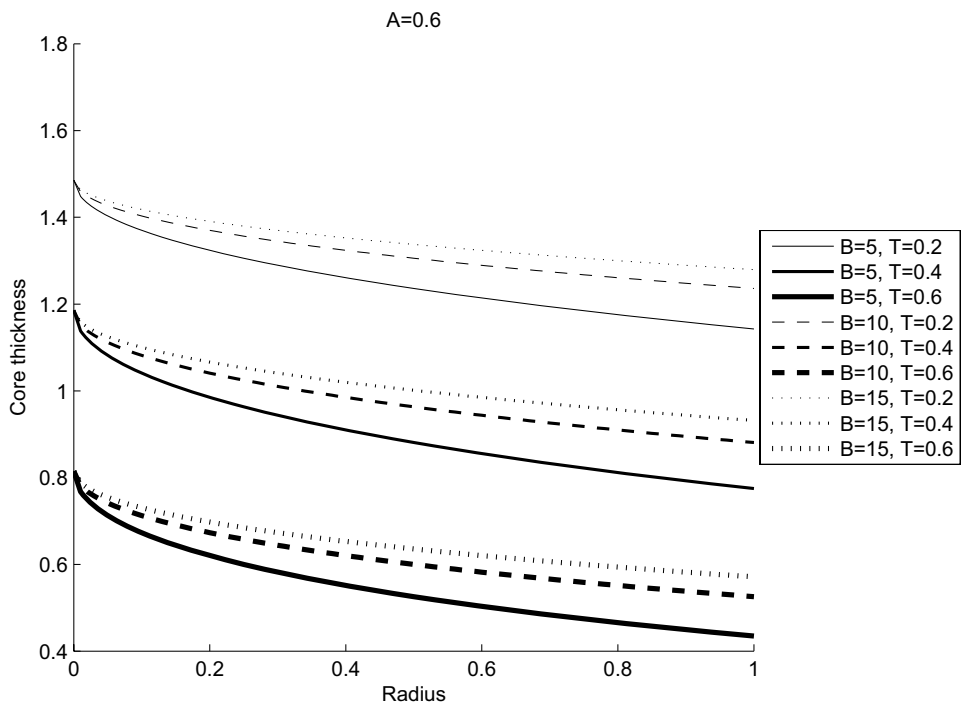

Figure 5 - Core thickness variation along the radius for $A=0.6$.

increasing with the increase of the non-Newtonian characteristic $B$. In limiting case as $B$ tends to zero, the velocity profile is that of a Parabola, which represents a newtonian fluid. 


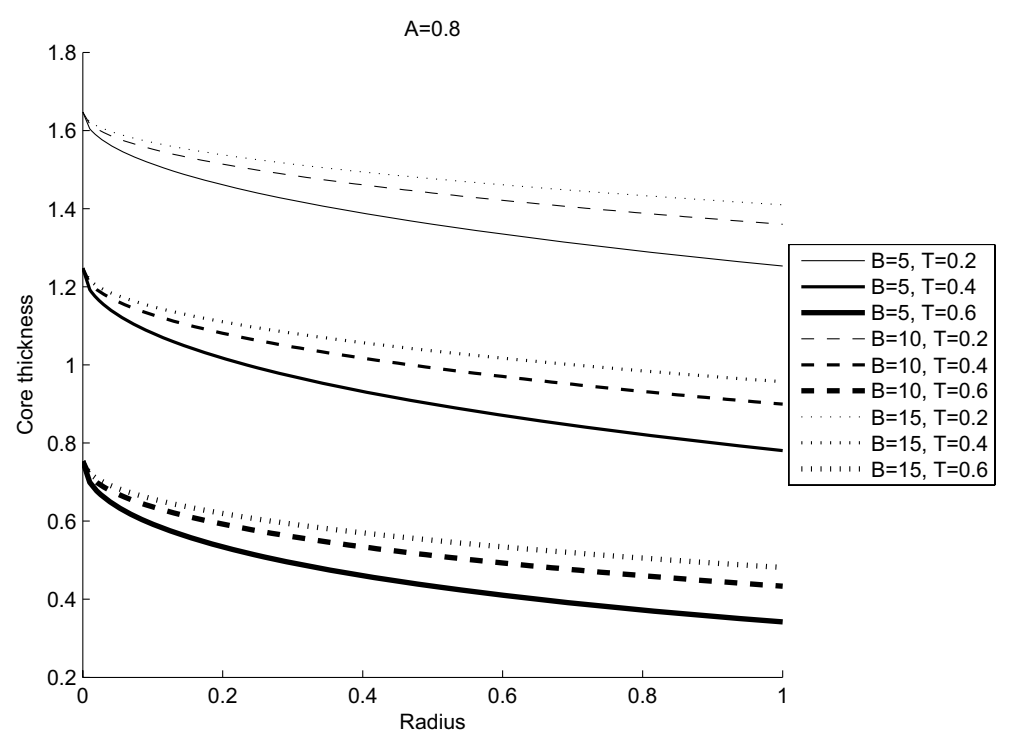

Figure 6 - Core thickness variation along the radius for $A=0.8$.

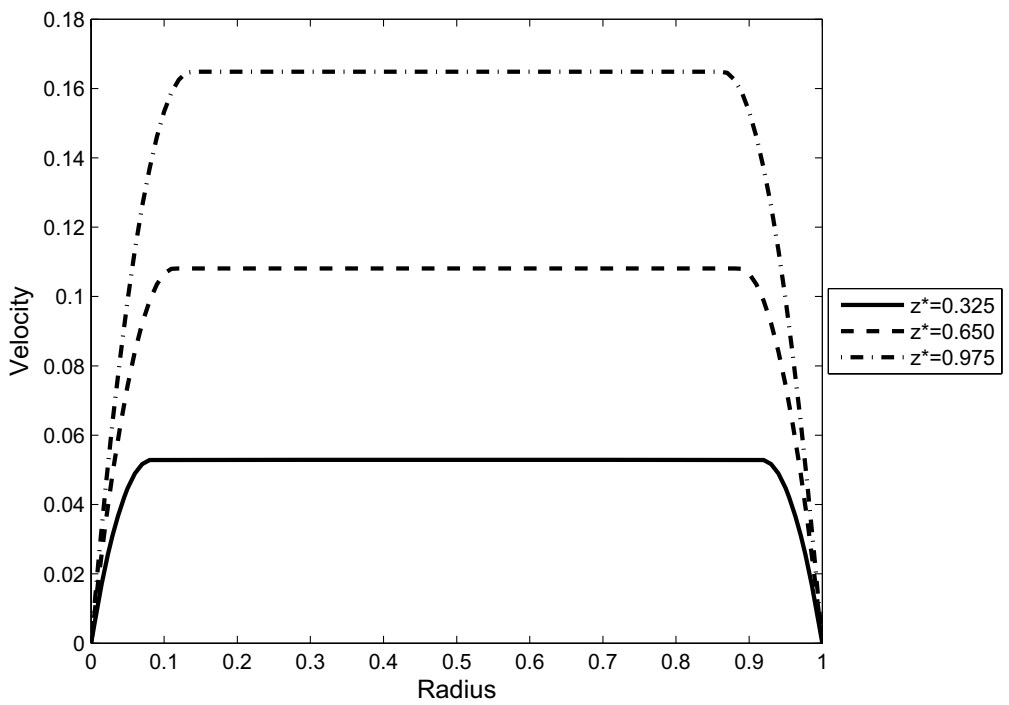

Figure 7 - Velocity profile for $A=0.6, T=0.3333, B=5$.

The results of load capacity calculated for various values of Reynolds number, Bingham number, time and amplitudes are graphically shown in the Figs. 1215. We find that the load capacity of the bearing increases as Reynolds number 


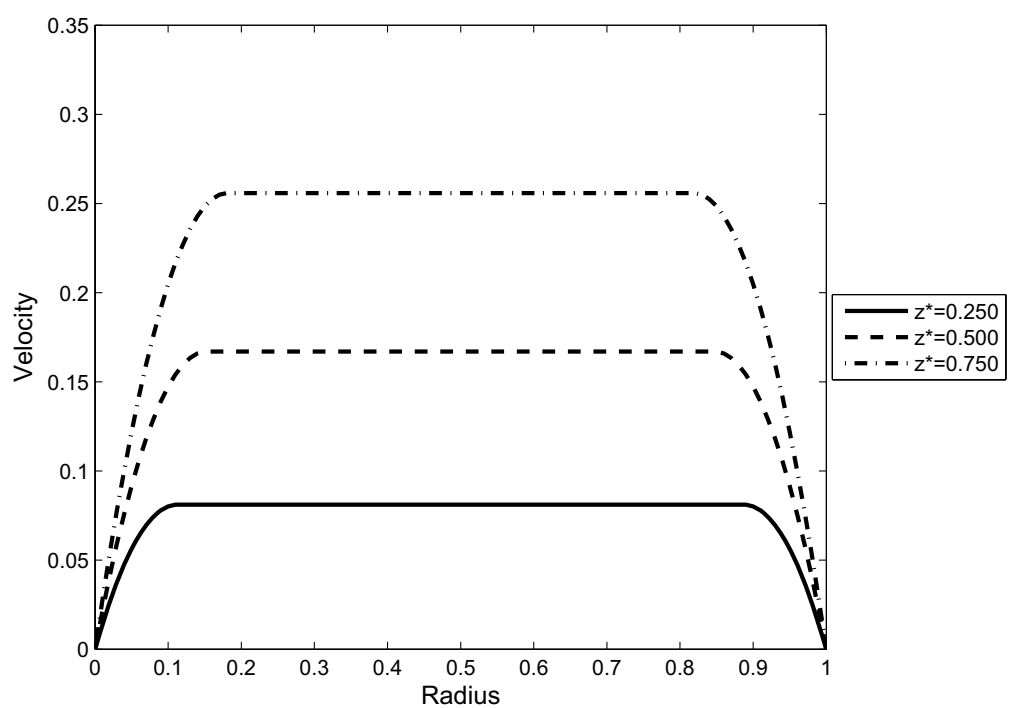

Figure 8 - Velocity profile for $A=0.6, T=0.5000, B=5$.

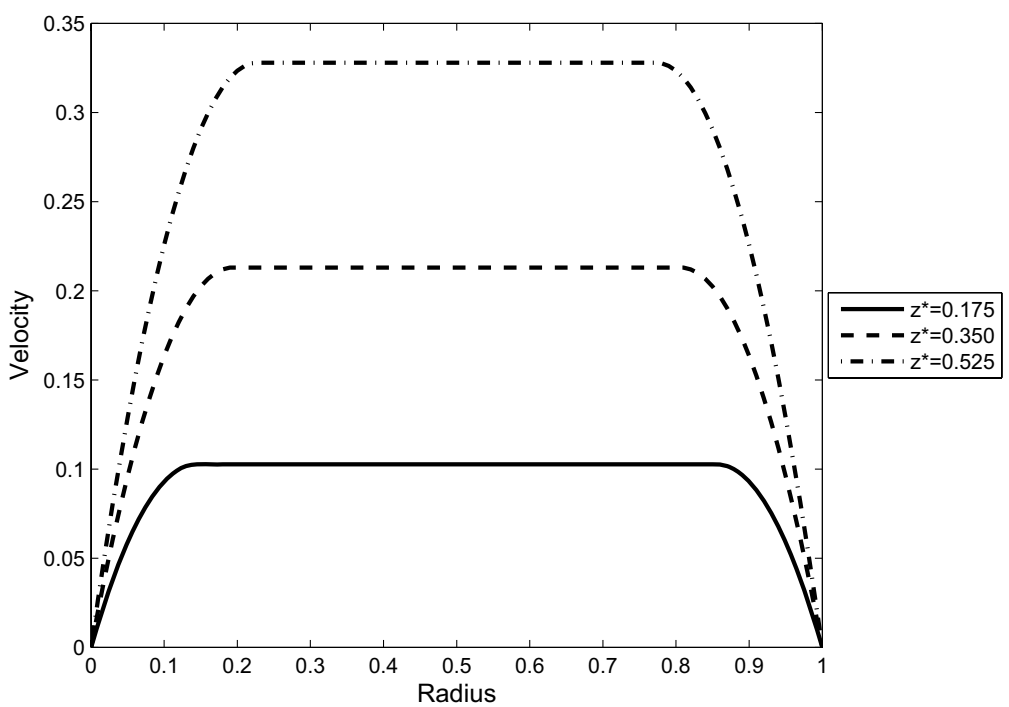

Figure 9 - Velocity profile for $A=0.6, T=0.6667, B=5$.

increases for a particular Bingham fluid at a particular amplitude but the rate of increase of the load capacity due to inertia is found to be less for fluids with high Bingham number. This means that the effect of inertia is small for high 


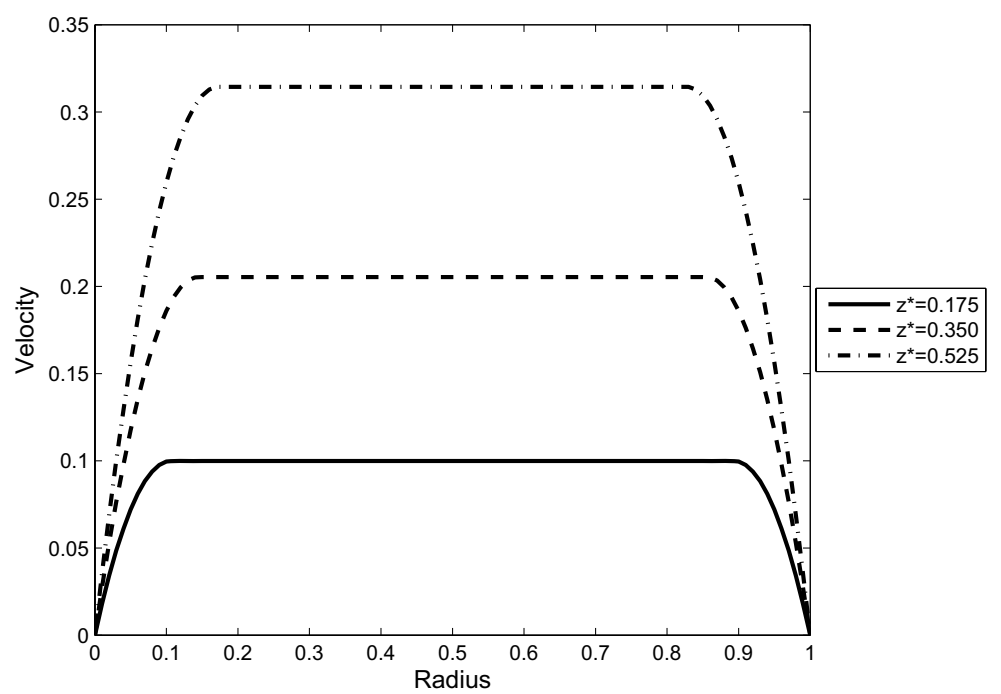

Figure 10 - Velocity profile for $A=0.6, T=0.6667, B=10$.

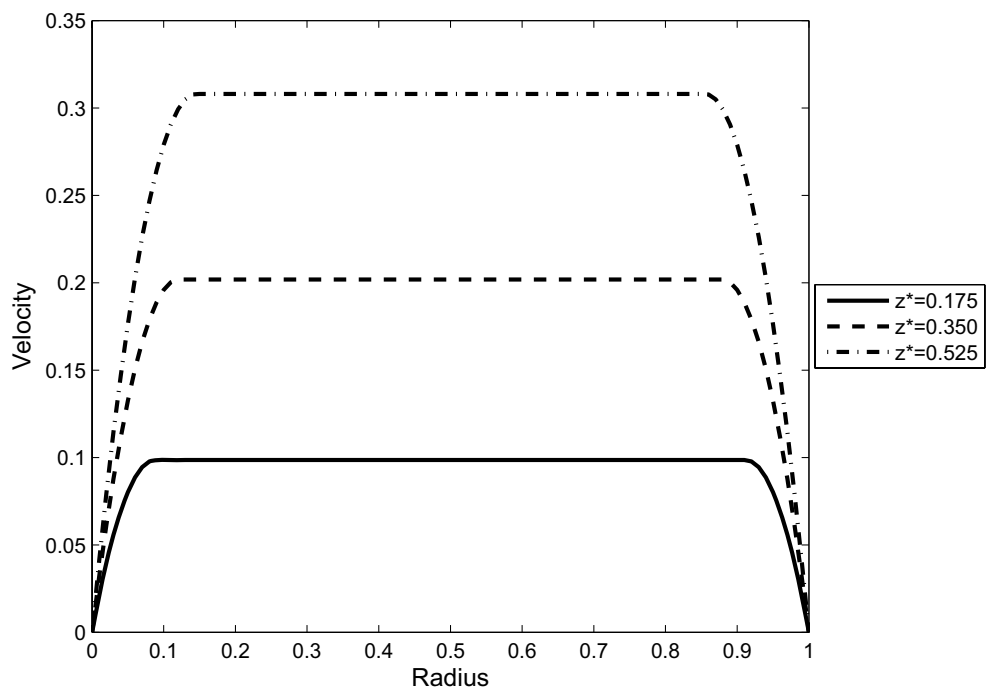

Figure 11 - Velocity profile for $A=0.6, T=0.6667, B=15$.

non-Newtonian characteristic values. Moreover, for a constant amplitude and Bingham number the load capacity of the bearing increases with the increase of time up to an optimum point, beyond which it starts decreasing with further increase in time. The effects of both inertia forces and non-Newtonian charac- 


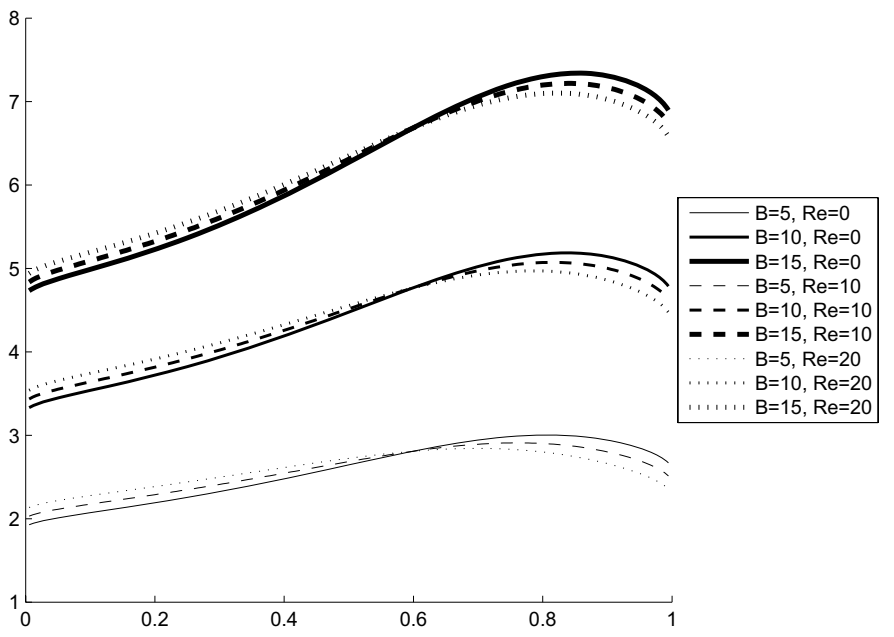

Figure 12 - Load capacity variation with time for $A=0.2$.

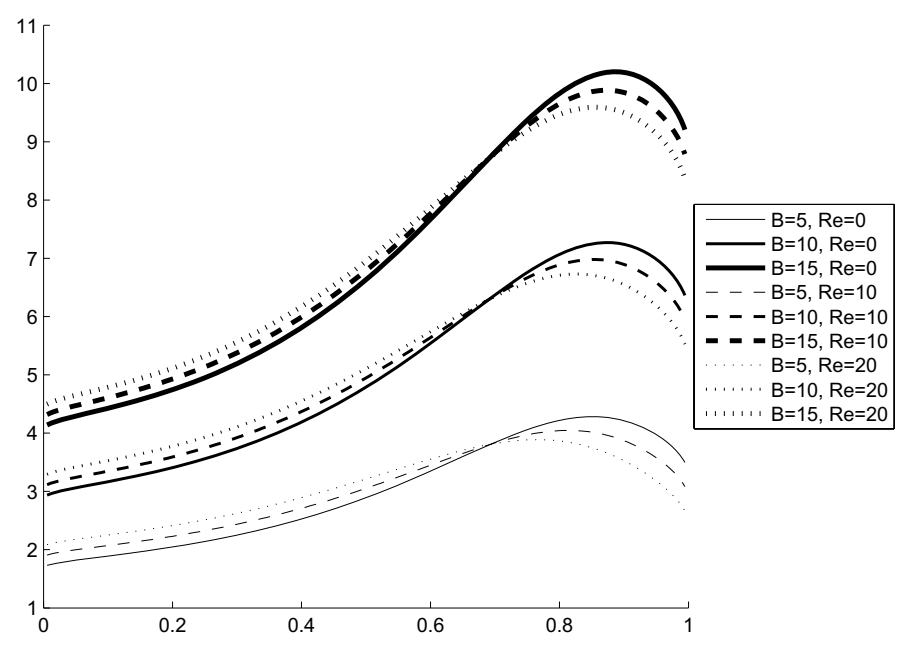

Figure 13 - Load capacity variation with time for $A=0.4$.

teristics on the load capacity are more significant as the amplitude of the squeeze motion increases.

To the best of our knowledge, no work is available for squeeze film bearing under sinusoidal motion with time-independent non-Newtonian fluids having yield stress. For the case of constant squeeze motion, the present results are found to be in agreement with the results obtained by Batra and Kandasamy [1]. 


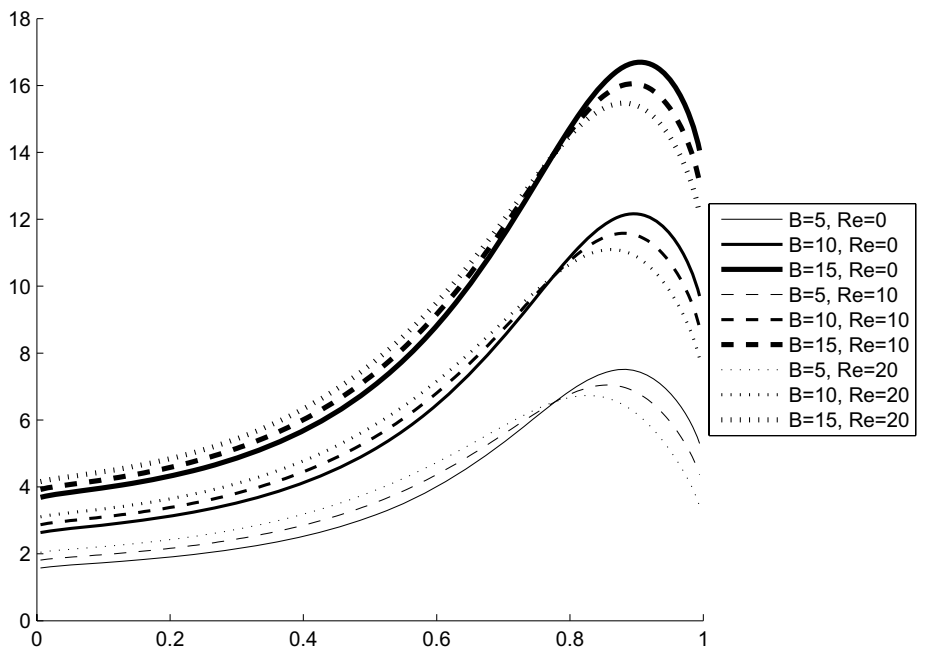

Figure 14 - Load capacity variation with time for $A=0.6$.

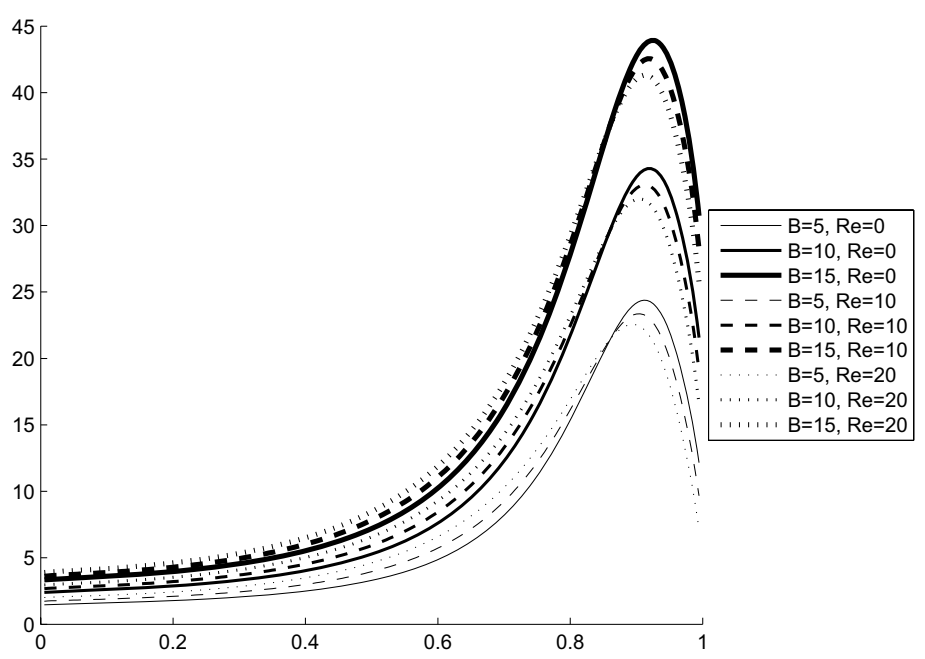

Figure 15 - Load capacity variation with time for $A=0.8$.

Acknowledgement. The authors would like to thank the reviewers for their valuable comments.

\section{REFERENCES}

[1] R.L. Batra and A. Kandasamy, Inertia effects in rheodynamic lubrication of a squeeze film bearing. Wear, 131 (1989), 273-282. 
[2] G.H. Covey and B.R. Stanmore, Use of parallel-plate plastomer for the characterization of viscous study with a yield stress. J. Non-Newtonian Fluid Mech., 8 (1981), 249-260.

[3] A.F. Elkouh, Fluid inertia effects in non-Newtonian squeeze films. ASME Trans. J. Lub. Tech., 98 (1976), 409-411.

[4] D.K. Gartling and N. Phan-Thien, A numerical simulation of a plastic flow fluid in a parallel plate plastomer. J. Non-Newtonian Fluid Mech., 14 (1984), 347-360.

[5] H. Hashimoto and S. Wada, The effects of Fluid Inertia Forces in Squeeze Film Bearings Lubricated with Pseudo-plastic Fluids. Bulletin of JSME, 29 (1986), 1913-1918.

[6] D.C. Huang, B.C. Liu and T.Q. Jiang, An analytical solution of radial flow of a Bingham fluid between two fixed circular disks. J. Non-Newtonian Fluid Mech., 26 (1987), 143-148.

[7] E.J. O'Donovan and R.I. Tanner, Numerical study of the Bingham Squeeze film problem. J. Non-Newtonian Fluid Mech., 15 (1984), 75-83.

[8] J.A. Tichy and W.O. Winer, Inertial considerations in parallel circular Squeeze film bearings. ASME Trans. J. Lub. Tech., 92(4) (1970), 588-592.

[9] R. Usha and P. Vimala, Inertia effects in circular squeeze films containing a central air bubble. Fluid Dynamics Research, 26 (2000), 149-155.

[10] R. Usha and P. Vimala, Curved squeeze film with inertial effects-energy integral approach. Fluid Dynamics Research, 30 (2002), 139-153. 